المجلة الدولية للدراسات التربوية والنفسية

بحث رقم 5

المجلد العاشر، العدد الأول، 101:94

(2021) (1آب

\title{
دور المنصة الرقمية "سفير" في التخفيف من الاغتراب النفسي لدى الطلبة المبتعثين في الولايات المتحدة الأمريكية
}

\author{
تماضر منصور سالم المعاشي
}

جامعة حائل- المملكة العربية السعودية deaa34767@gmail.com

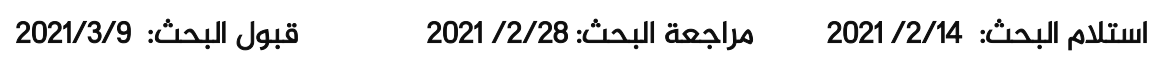

DOI: https://doi.org/10.31559/EPS2021.10.1.5

(1) file is licensed under a Creative Commons Attribution 4.0 International 


\title{
دور المنصية الرقمية "سفير" في التخفيف من الاغتراب النفسي لدى الطلبة المبتعثين في الولايات المتحددة الأمريكية
}

\author{
تماضر منصور سالم المعاشي \\ جامعة حائل- المملكة العربية السعودية \\ deaa34767@gmail.com
}

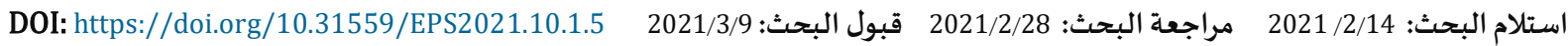

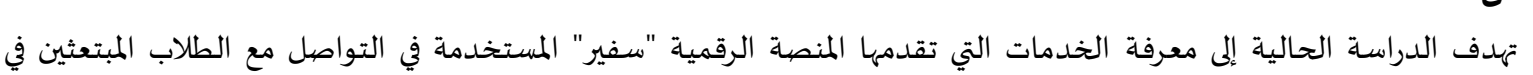

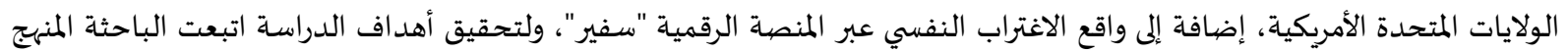

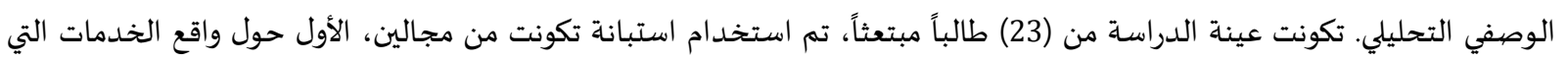

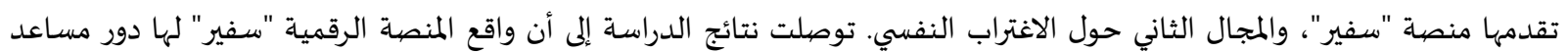

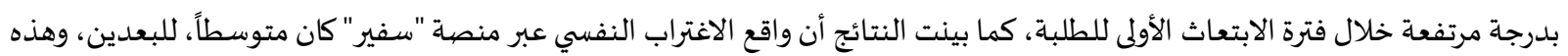

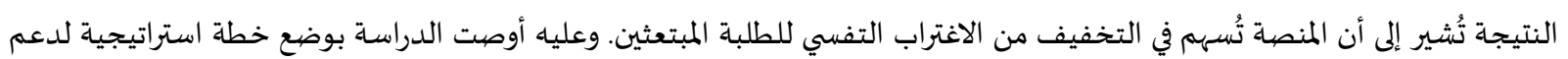

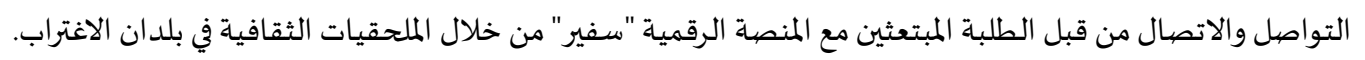

$$
\text { الكلمات المفتاحية: المنصة الرقمية "سفير"؛ الاغتراب النفسي؛ الابتعاث. }
$$

أصبح من المعلوم أن العملية التعليمية برمتها تأثرت بالتطور التقني والتغيرات السريعة الناجمة عن التقدم العلمي وتقنيات المعلومات، إذ ظهرت

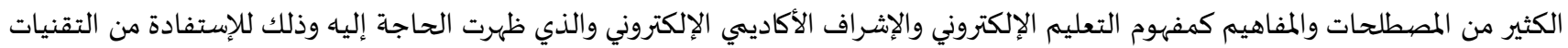

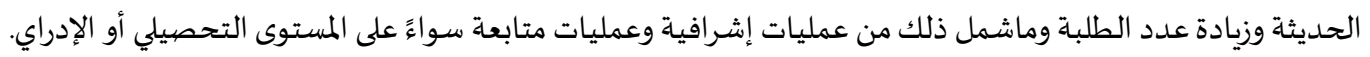

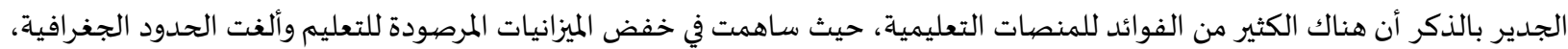

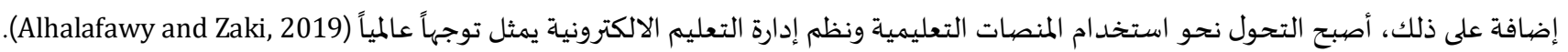

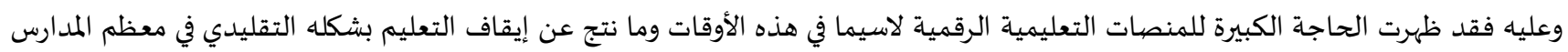

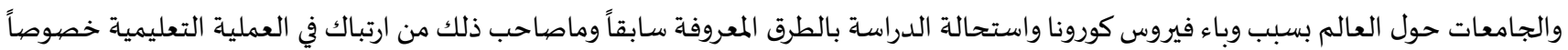

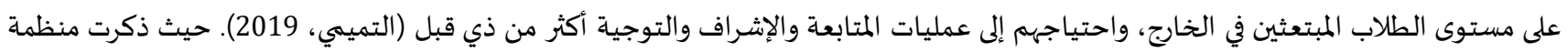
اليونيسكو في تقريرها الصادر في 2020/03/26م، إلى تأثر 87\% من عدد الطلاب حول العالم بإغلاق مدارسهم وجامعاتهم (Hofhuis and al, 2019).

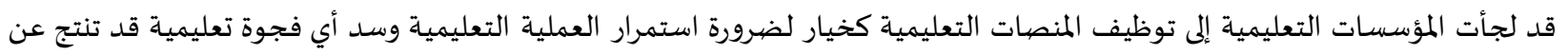

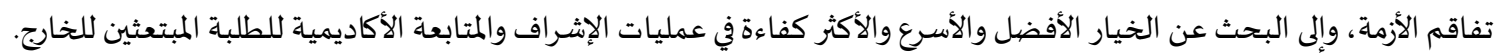

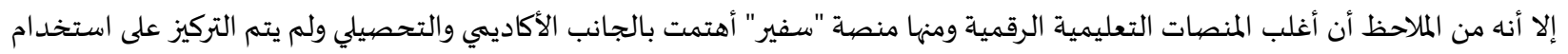
هذه المنصات كوسيلة وكقناة إتصال وتواصل مع الطلبة المبتعثين لمساعدتهم على حل الإشكالات التي قد تواجههم في بلد الابتعاث (الغامدي، 2016). 
وما قد يصاحبها من عدم التكيف وشعور الطلبة بالوحدة، والعزلة الناتجة عن الغربة في بلد الابتعاث، لاسيما في ظل حداثة هذه التجربة على جميع الطلبة (جدوع، 2019).

مع الزيادة الملحوظة في أعداد الطلبة بالخارج ، ظهرت الحاجة الماسـة لبناء نظام متكامل لاحتواء هذا الكم الهائل من البيانات من أجل الانتقال إلى

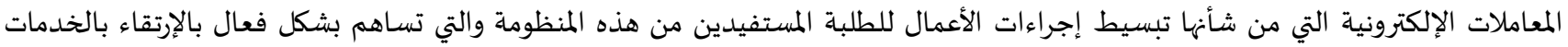

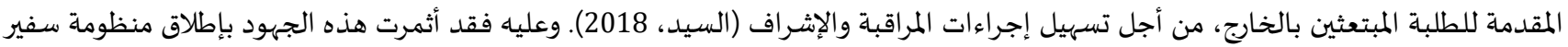

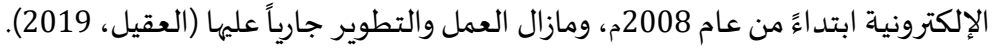

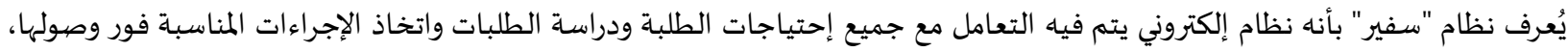

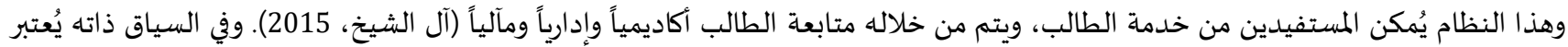

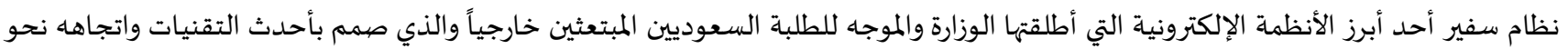

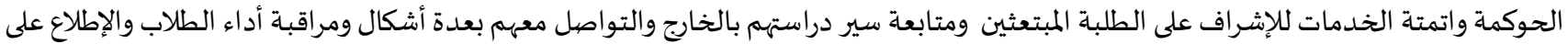
التقارير الدورية لتحصيل الطلاب ومساعدة الطلاب على حل جميع التحديات التي تواجههم (الشريف، 2020). وتجدر الإشـارة إلى أن المنصية التعليمية "سفير" تُعتبر من أهم الإبتكارات التكنولوجية التي زودت الطلبة المبتعثين بالمزايا والخصائص والخدمات التي سهلت العملية التعليمية (النعيم، 2015).

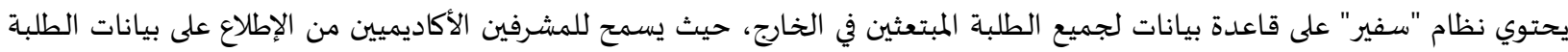

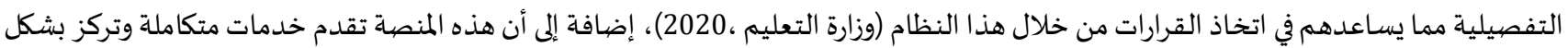
خاص على تقديم الدعم الإلكتروني والمحتوى لزيادة التواصل والاتصال في بلدان الابتعاث ( الحلفاوي، 2017).

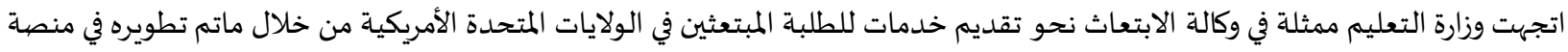

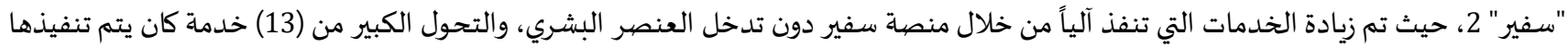

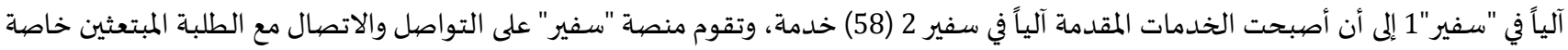
فيما يختص بالجوانب الاجتماعية والجوانب النفسية، إضافة إلى رسائل الدعم والإرشاد والتوجيه للطلاب لمساعدتهم في تجاوز ما يواجههونه في بلدان

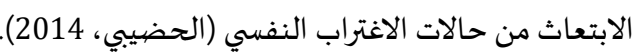
وأمام كل هذه التحديات توجد نسبة من الطلاب يواجهون بعض الصعوبات المتعلقة بعدم التكيف داخل مؤسساتهم التعليمية وشعورهم

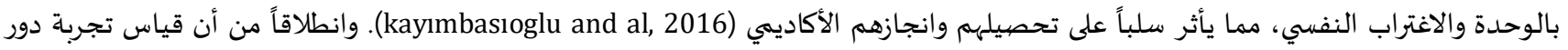

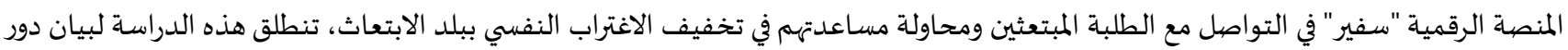
منصية "سفير" في التخفيف من الاغتراب النفسي لدى الطلبة المبتعثين في الولايات المتحدة الأمريكية.

1.1

انطلقت الدراسـة من بعض تحليل المشاكل التي تواجه طلبة الابتعاث في الولايات المتحدة الأمريكية، حيث تبين هذا من خلال التقارير الواردة من

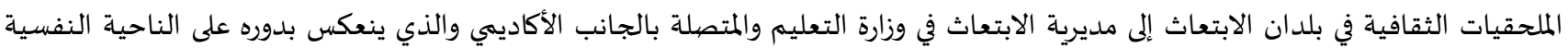
للطلبة المبتعثين، حيث يواجهوان مجموعة تحديات منها ما يتصل بثقافة بلد الابتعاث، إضافة إلى اللغة، وما يتصل أيضاً بطرق التكيف الاجتمائي

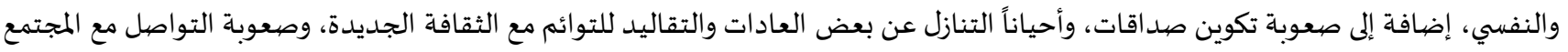
الجديد، حيث يمتد تأثير هذه المشكلات إلى منح الطلبة شعوراً بالاغتراب النفسي وهو ما يمنحهم الإحساس بالعزلة وفقدان الثقة بالنفس، وهذا كله

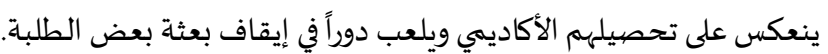

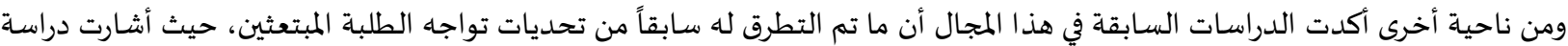
الرويلي (2019) إلى بعض معوقات إتمام بعثة أعضياء هيئة التدريس السعوديين في الجامعات الأجنبية، كما بينت دراسة عقيل (2017) تجربة الدراسة بالخارج وعلاقتها بالذكاء الثقافي لدى مبتعثي التربية الخاصة من المملكة العربية السعودية، ودراسة الأسمري (2019) لبيان الصددمة الثقافية لدى لدئ

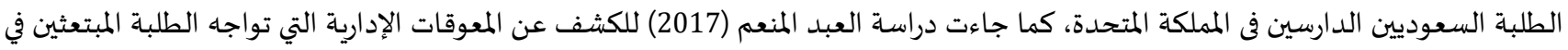
دولتي أمريكا والمملكة المتحدة. وفي إطار ذلك تتمثل مشكلة الدراسة بالتساؤل الرئيس التالي: ما دور المنصية الرقمية "سفير" في التخفيف من الاغتراب النفسي لدى الطلبة المبتعثين في الولايات المتحدة الأمريكية؟

ما فاعلية المنصية الرقمية "سفير" المستخدمة في التواصل مع الطلاب المبتعثين في الولايات المتحدة الأمريكية؟ ما واقع الاغتراب النفسي عبر المنصة الرقمية "سفير" المستخدمة في التواصل مع الطلاب المبتعثين في الولايات المتحدة الأمريكية؟ 


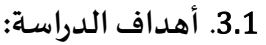

التعرف إلى الخدمات التي تقدمها المنصاة الرقمية "سفير" المستخدمة في التواصل مع الطلاب المبتعثين في الولايات المتحدة الأمريكية؟ التعرف إلى واقع الاغتراب النفسي عبر المنصية الرقمية "سفير" المستخدمة في التواصل مع الطلاب المبتعثين في الولايات المتحدة الأمريكية؟

4.1 - M أهمية الدراسـة:

تبرز أهمية الدراسة فيما يلي:

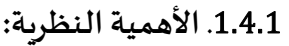

إلقاء الضوء على الخدمات التي تقدمها المنصة الرقمية "سفير" للطلبة المبتعثين في الولايات المتحدة الأمريكية؟ إلقاء الضوء على واقع الاغتراب النفسي عبر المنصية الرقمية "سفير" للطلبة المبتعثين في الولايات المتحدة الأمريكية. إلقاء الضوء على مؤشرات مؤشرات الاغتراب النفسي عبر المنصة الرقمية "سفير".

2.4.1 الأهمية التطبيقية:

الكشف عن التحديات الناتجة عن الصدمة الثقافية لدى الطلبة المبتعثين في الولايات المتحدة الأمريكية.

قد تُسهم الدراسـة الحالية في بيان أساليب التكيف النفسي والإجتماعي التي من الواجب توظيفها للتخفيف من الاغتراب النفسي لدى الطلبة المبتعثين.

من المتوقع أن تسهم نتائج هذه الدراسة في إفادة متخذي القرار، وواضعي السياسات في وزارة التعليم ومكتب الابتعاث بالنظر إلى الطرق والأساليب الفعالة في التخفيف من المعوقات التي تواجه الطلبة المبتعثين.

5.1. حدود الدراسـة:

•الحد الموضيوعي: تتمثل في الكشف عن دور المنصة الرقمية "سفير" للتخفيف من الاغتراب النفسي للطلبة المبتعثين في الولايات المتحدة

الأمريكية.

• • الحدود المكاني والزماني: الولايات المتحدة الأمريكية للعام الدراسي 2021.

• الحد البشري: الطلاب السعوديون المبتعثون للولايات المتحدة الأمريكية.

6.1.

الاغتراب النفسي: هو شعور الفرد بالانفصال عن الآخرين أو عن الذات أو كليهما، وبهذا المعنى فالاغتراب النفسي يتمثل في العجز، وعدم الالتزام بالمعايير، وفقدان المعنى والتمحور حول الذات وعدم الإحساس بالقيمة الاجتماعية والنفسية للذات (Alosaimi et al, 2016). وتُعرفه الباحثة

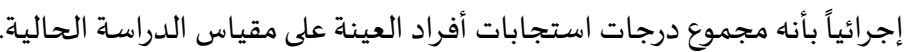

ه المنصية الرقمية "سفير": هي نظام إلكتروني يتم فيه التعامل مع جميع إحتياجات الطلبة، ويتم من خلاله متابعة الطلبة أكاديمياً وإدارياً ومآلياً.

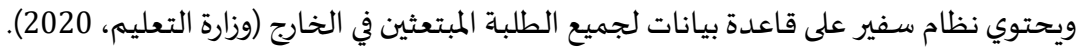

2. إجراءات الدراسـة الميدانية

1.2.

اتبعت الدراسة الحالية المنهج الوصفي التحليلي، حيث يتم من خلاله دراسة الظاهرة كما هي في الواقع ويصفها وصفاً دقيقاً.

2.2. تينة الدراسـة

تمثلت عينة الدراسة بعينة قصدية بلغت (23) طالباً، وتم الحصول على بياناتهم من وكالة الابتعاث في وزارة التعليم، حيث تم التواصل معهم من

خلال Email \& Whatsapp

3.2.

تم استخدام استبانة تكونت من مجالين، الأول حول واقع الخدمات التي تقدهها منصة "سفير"، والمجال الثاني تركز على حول الاغتراب النفسي

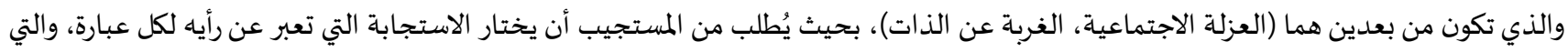

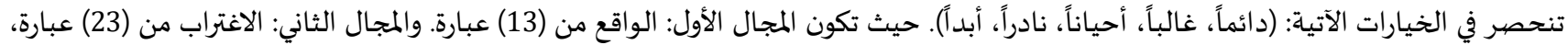

وقد استفادت الباحثة من دراسة (كباجة، 2015). 
4.2 صهدق أداة الدراسة (الاستبانة):

تم التحقق من صهدق الاستبانة عن طريق صدق الاتساق الداخلي، والجدول التالي يبين ذلك

\begin{tabular}{|c|c|c|}
\hline قيمة الدلالة & معامل الارتباط & المجال \\
\hline 0.000 & ${ }^{* *} 0.73$ & الاغتراب النفسي \\
\hline 0.000 & $* * 0,84$ & واقع الخدمات \\
\hline
\end{tabular}

يتبين من الجدول (1) أن معاملات ارتباط بيرسون عالية، كما أهها دالة إحصائياً عند مستوى دلالة (0.01)، وهذا يدل على أن جميع هذه المجالات تتمتع بصداق الاتساق الداخلي.

5.2. ثبات أداة الدراسة (الاستبانة): اتبعت الباحثة طريقة ألفا كرو نباخ Cronbach's Alpha ويُظهر الجدول التالي معاملات الثبات. جدول (2): معاملات ألفا كرونباخ للثبات

\begin{tabular}{|c|c|c|c|}
\hline معامل ألفا كرو نباخ & عدد البنود & المحاور الفرعية & 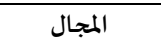 \\
\hline 0.708 & 7 & العزلة الاجتماعية & الاغتراب النفسي \\
\hline 0.693 & 7 & الغربة عن الذات & \\
\hline 0.761 & 6 & & ع الخدمات \\
\hline
\end{tabular}

يتضح من الجدول أن معاملات ألفا كرونباخ هي معاملات ثبات عالية، ويمكن الاعتماد عليها في التطبيق الميداني للدراسة.

3. نتائج الدراسة ومناقشتها

1.3. السؤال الأول: ما فاعلية المنصية الرقمية "سفير" المستخدمة في التواصل مع الطلاب المبتعثين في الولايات المتحدة الأمريكية من وجهة نظرهم؟ تم حساب المتوسطات الحسابية والانحرافات المعيارية، للخدمات التي تقدمها المنصية الرقمية "سفير" كما موضيح في الجدول التالي:

\begin{tabular}{|c|c|c|c|c|c|}
\hline درجة الو اقع & الترتيب & 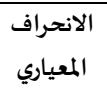 & الحسابي & 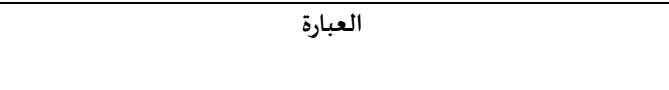 & م \\
\hline 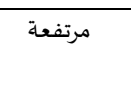 & 1 & 0.738 & 4.107 & الطنلة فهيها منهية "سفير" ورش العمل من خلال أدواتها على النظام وتُشرك & 5 \\
\hline 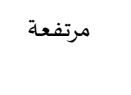 & 2 & 0.842 & 4.035 & تُسهم منصة "سفير" توفير المعلومات للطلبة المبتعثين عن قوانيين بلد & 2 \\
\hline 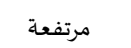 & 3 & 0.709 & 3.892 & تعزز منصة "سفير" ارتباط المبتعث بهويته السعودية. & 6 \\
\hline مرتفعة & 4 & 1.011 & 3.821 & توفر منصة "سفير" بيانات وأدوات لحل مشاكل الطلبة المبتعثين. & 1 \\
\hline 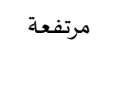 & 5 & 1.037 & 3.714 & تالابتعاث. منصة "سفير" الفعاليات والأنشطة الأكاديمية للطلبة المبتعثين في بلد & 4 \\
\hline 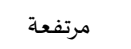 & 6 & 1.183 & 3.261 & توفر منصة "سفير" الدعم الفني للمشاكل التقنية التي تواجه المبتعثين. & 3 \\
\hline 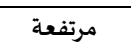 & & 0.782 & 3.805 & المتوسط الموزون & \\
\hline
\end{tabular}

يوضح الجدول (3) قيم المتوسطات الحسـابية لواقع المنصة الرقمية "سفير" المستخدمة في التواصل مع الطلاب المبتعثين في الولايات المتحدة الأمريكية، حيث تبين أن لها دور مساعد بدرجة مرتفع خلال فترة الابتعاث الأولى لهم، حيث بلغ المتوسط الحسابي للدرجة الكلية لهذه الخدمات المات (3.805)، وانحراف معياري قدره (0.782)، كما تراوحت انحرافاتها بين (0.709 - 1.183) وذلك يشير إلى انخفاض درجة التشتت بين استجابات عينة

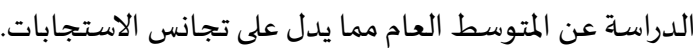
تُفسر هذه النتيجة إلى أن الطلبة المبتعثين إلى الولايات المتحدة الأمريكية في بداية فترة ابتعاثهم يهتمون بمتبعة الأخبار والمستجدات من الناحياة

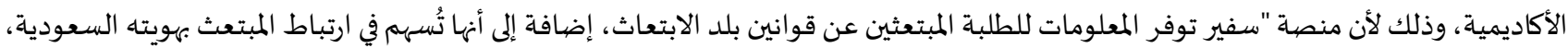
وهذا يبين الأثر لمنصاة سفير على واقع وحياة المبتعث في بلد الابتعاث، كما أن ورش العمل لها دوراً في حياة المبتعث، حيث يتم إشراكه بها لتعريفه

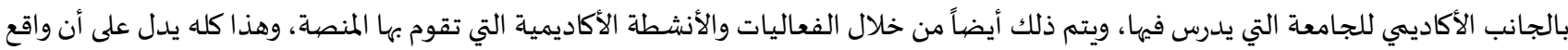
الخدمات التي تقدمها المنصة تلعب دوراً مهماً في حياة الطالب المبتعث من الناحية الأكاديمية ومن الناحية الاجتماعية والنفسية في بلد الابتعاث. وجاءت هذه النتيجة مرتبطة مع ما أشارت إليه دراسة كل عقيل (2017) تجربة الدراسة بالخارج وعلاقتها بالذكاء الثقافي لدى مبتعثي التربية الخاصية

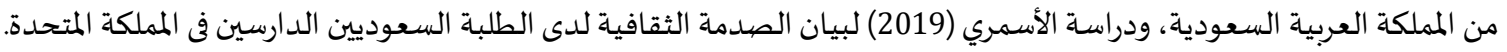


2.3. السؤال الثاني: ما و اقع الاغتراب النفسي عبر المنصية الرقمية "سفير" المستخدمة في التواصل مع الطلاب المبتعثين في الولايات المتحدة

الأمريكية؟

تم حساب المتوسطات الحسابية والانحرافات المعيارية، لواقع الاغتراب النفسي عبر المنصة الرقمية "سفير"، كما موضح في الجدول التالي:

\begin{tabular}{|c|c|c|c|c|c|}
\hline الترتيب & الدور & المعياري & المتسابي & 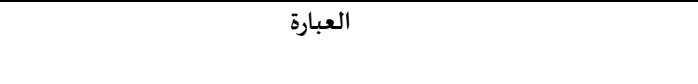 & م \\
\hline 1 & متوسط & 1.209 & 3.610 & ينتابني حالات من الاكتئاب عندما اتعامل مع الآخرين في بلد الابتعاث & 4 \\
\hline 2 & متوسط & 1.271 & 3.518 & لا استطيع الاندماج في البيئة الجامعية في بلد الابتعاث. & 6 \\
\hline 3 & متوسط & 1.433 & 3.381 & أشعر بأنه ليس لدي أي طموح أو هدف أسعى لتحقيقه في فترة ابتعاثي. & 3 \\
\hline 4 & 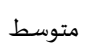 & 1.071 & 3.202 & الشعور بالتناقض بين ما أرغب بتحقيه وبين ما يريده الآخرون في بلد الابتعاث. & 1 \\
\hline 5 & متوسط & 1.046 & 3.285 & أشعر بأني غير قادر على اتخاذ قرارات فيما يخص حياتي في بلد الابتعاث. & 5 \\
\hline 6 & 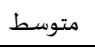 & 0.720 & 3.182 & أحس بالحنين والاشتياق لأهلي وأصدقائي في بلدي الأم خلال فترة ابتعاثي. & 2 \\
\hline \multicolumn{2}{|c|}{ متوسط } & 0.729 & 3.363 & المتوسط الموزون للعجزوالعزلة الاجتماعية & \\
\hline 1 & متوسط & 0.491 & 3.520 & لا اشعر بالرضا عن دراستي في بلد الابتعاث، بحيث أشعر أن لا قيمة لي. & 1 \\
\hline 2 & متوسط & 1.386 & 3.408 & 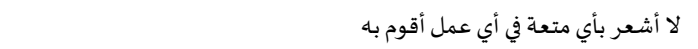 & 2 \\
\hline 3 & متوسط & 1.391 & 3.371 & لم يحقق لي الواقع قي بلد الابتعاث أية رغبة من رغباتي & 3 \\
\hline 4 & متوسط & 1.442 & 3.301 & لا أستطيع التعبير عما في داخلي، بحيث أشعر بغربة وعجز تام في بلد الابتعاث. & 4 \\
\hline 5 & متوسط & 0.702 & 3.261 & دائماً أشعر بالعناء والحزن في بلد الابتعاث. . & 5 \\
\hline 6 & متوسط & 0.629 & 3.108 & أشعر بأن مستقبلي مجهول وأفكر بالفشل في دراستي في بلد الابتعاث. & 6 \\
\hline \multicolumn{2}{|c|}{ متوسط } & 0.832 & 3.328 & المتوسط الموزون للغربة عن الذات & \\
\hline
\end{tabular}

يتبين من النتائج الوارة في الجدول (4) أن قيم المتوسطات الحسابية لواقع الاغتراب النفسي عبر منصة "سفير" كان متوسطاً، للبعدين، حيث جاء

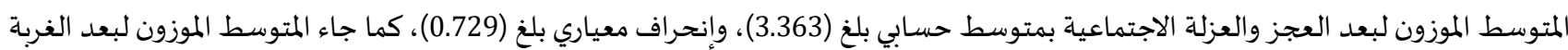

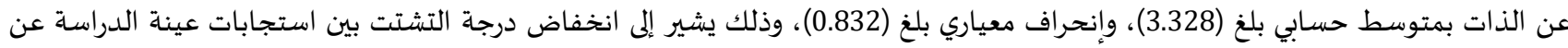
المتوسط مما يدل على تجانس الاستجابات. وترجح الباحثة هذه النتيجة إلى أن هناك دوراً متوسطاً لمنصاة سفير في خفض الاغتراب النفسي لدى الطلبة المبتعثين، حيث أن وجود الطلبة في

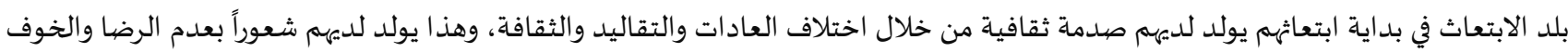

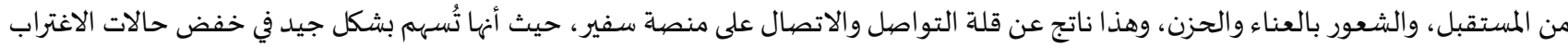
النفسي لدى الطلبة المبتعثين، وأن هذا الدور يتضح من خلال ما تقوم به المنصية من تواصل واتصال مع الطلبة حتى تخرجهه من العزلة في بلد الابتعاث. وبالنتيجة فمنصة سفير تلعب دوراً بأخراج المبتعث من دائرة الأحداث الضاغطه التي يواجهونها في بلد الابتعاث. وجاءت هذه النتيجة مرتبطة مع ما أشـارت إليه دراسـة كل من الرويلي (2019) حول معوقات إتمام بعثة أعضاء هيئة التدريس السعوديين في الجامعات الأجنبية، و دراسة عقيل

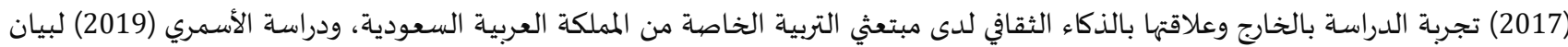

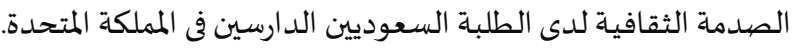

3.3. توصيات الدراسـة:

في ضوء النتائج التي تولدت عن الدراسة التطبيقية، أوصت الباحثة بمحموعة من التوصيات يمكن إجمالها بالتالي: وضع خطة استراتيجية لدعم التواصل والاتصال من قبل الطلبة المبتعثين مع المنصبة الرقمية "سفير" من خلال الملحقيات الثقافية في بلدان

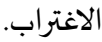

وضع خطة من قبل وكالة الابتعاث في وزارة التعليم حول تطوير وتحسين أدوات "سفير" وتحسينها إلى "سفير" 3 حتى يتم تشجيع طلبة الابتعاث على التواصل والاتصال مع هذه المنصية. بناء برنامج تدريبي، إضافة إلى الفعاليات والأنشطة التي من شأنها تبين مميزات أدوات منصة "سفير" و "سفير" 1، و"سفير" 2 ، وإطلاع الطلبة المبتعثين عن أي تطوير وتحديث على المنصية. النظر إلى الجانب الأكاديمي للطلبة المبتعثين، بحيث من خلاله يتم تحديد مدى اندماجهم في المجتمع الجديد، وهذا يعطي دلالة على تكيفهم النفسي في حال كان الجانب الأكاديمي جيداً. النظر في مكونات الاغتراب النفسي لدى الطلبة المبتعثين وإيجاد الحلول المناسبة لتفادي التحديات التي تواجههم في بلد الابتعاث. 


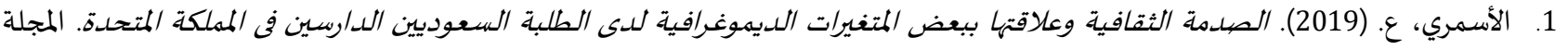

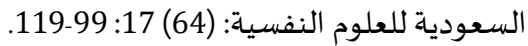

2. التميهي، ن. (2019). المشكلات التي تواجه طلاب الدراسيات العليا المبتعثين للخارج بجامعة الأمير سطام بن عبد العزيز. مجلة العلوم التربوية:

.474-419:(18)

3. جدوع، هـ. (2019). دور شبكات التواصل الاجتماعي في تشكيل معارف وإتجاهات المبتعثين السعوديين تجاه القضايا المحلية. المجلة العربية

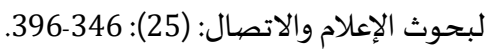

4. الحضيبي، أ. (2014). المشككلات التي تواجه الطلبة السعبديين المبتعثين إلى الولايات المتحدة الأمريكية ، مجلة جامعة شقراء: (2) 4: 101-135. 5. الحلفاوي، و. (2017). نموذج مقترح لمنصية فنية عبر الويب وقياس فاعليتها في تنمية التفكير الإبلاعي لدى الطابلاب المعلمين في التوبية الفنية.

المجلة المصرية للدراسات المتخصصية: (15) 8: 51-715.

6. الرويلي، أ. (2019). معوقات إتمام بعثة أعضاء هيئة التدريس السعوديين في الجامعات الأجنبية. مجلة شؤون اجتماعية: (36): 9-30.

7. السيد، م. (2018). استخدام شبكات التواصل الاجتماعي وعلاقته بالاغتراب النفسي للدى طالبات جامعة طيبة. مجلة العلوم التربوية: (26) 3:

$.577-526$

8. الشريف، ش. (2020). واقع اتجاهات طلبة الجامعة نحو توظيف المنصيات الرقمية في التعليم الجامعي بالمملكة العببية السعودية. مجلة جامعة طيبة للآداب والعلوم الإنسانية: (22):

9. آل الشيخ، ن. (2015). عوامل تكيف الطالبة المبتعثة: دراسة تطبيقية على المبتعثات في الولايات المتحدة الأميكية. المجلة الاجتماعية: (10): 51.106

10. العبد المنعم، ف. (2017). المعوقات الإد/رية التي تواجه الطلاب المبتعثين في دولتي الولايات المتحدة الأمريكية والمملكة المتحدة والسبل المقترحة للتغلب عليها ، مجلة دراسات تربوية ونفسية: (94):

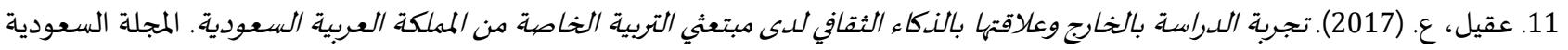
للتربية الخاصة: (3) 1: 65-100.

12. العقيل، ص. (2019). المخاطر الأمنية المحتملة التى قلد يتعرض لها المبتعثون السعوديون خارج المملكة وسبل الوقاية منها: دراسة على عينة من المبتعثين السعوديين في أمريكا وبريطانيا وأستراليا ، مجلة الدراسات الاجتماعية السعودية: المبكية (2): 58-88.

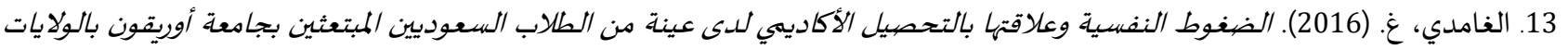

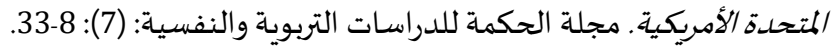
14. كباجة، س. (2015). التغير القيمي وعلاقتها بهوية الذات والاغتراب النفسي لدى طلبة الثانوية العامة في قطاع غزة. رسالة ماجستير غير منشورة، الجامعة الإسلامية بغزة. 15. النعيم، ع. (2015). العوامل التي ساعدت الفتيات على تفضيل الابتعاث إلى الدول المتقلدمة، دراسة مطبقة على المبتعثات السعوديات في

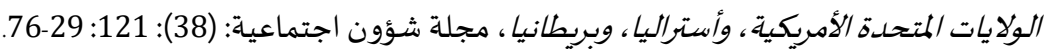
16. وزارة التعليم. (2020) . تم الإطلاع 1442/2/1 ، وتم إسترجاعه من المصددر:

ثانياً: المراجع الأجنبية:

1. Alhalafawy, W. S., \& Zaki, M. Z. (2019). The Effect of Mobile Digital Content Applications Based on Gamification in the Development of Psychological Well-Being. International Journal of Interactive Mobile Technologies (iJIM), $13(08)$ : $107-123$. https://doi.org/10.3991/ijim.v13i08.10725

2. Hofhuis, J., Hanke, K., \& Rutten, T. (2019). Social network sites and acculturation of international sojourners in the Netherlands: The mediating role of psychological alienation and online social support. International Journal of Intercultural Relations, 69: 120-130. https://doi.org/10.1016/j.ijintrel.2019.02.002

3. Kayımbasıoglu, D., Oktekin, B., \& Hacı, H. (2016). Integration of Gamification Technology in Education. Procedia Computer Science, 102: 668-676. https://doi.org/10.1016/j.procs.2016.09.460

4. Alosaimi , F. ; Alyahya , H. ; Alshahwan H. ; Almahyijari , N. \& Shaik , Sh.(2016). Smartphone addiction among university students in Riyadh, Saudi Arabia. Saudi Medical Journal, 37(6): 675-683. https://doi.org/10.15537/smj.2016.6.14430 
المجلة الدولية للدراسـات التربوية والنفسية

International Journal of Educational \& Psychological Studies (EPS)

Journal Homepage: https://www.refaad.com/views/EPSR/Home.aspx

www.refaad.com

ISSN: 2520-4149 (Online) 2520-4130 (Print)

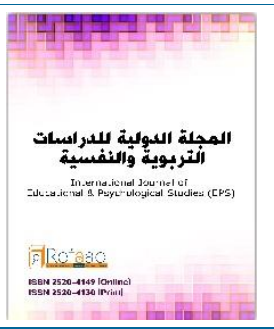

\title{
The role of the digital platform "Safeer" in alleviating the psychological alienation of students on scholarships in the United States of America
}

\author{
Tamaader mansor salem Almashe \\ University of Hail, KSA \\ deaa34767@gmail.com
}

Received: 14/2/2021 Revised : 28/2/2021 Accepted: 9/3/2021 DOI : https://doi.org/10.31559/EPS2021.10.1.5

Abstract: The study aim to identify the services provid by the digital platform "Safeer" used to communicate with students on scholarships in the United States of America. The study also aim to identify the reality of psychological alienation through the digital platform "Safeer". To achieve the objectives of the study, the researcher adopted the descriptive and analytical approach. The study sample consisted of (23) scholarship students. A questionnaire was used that consisted of two dimensions, the first about the reality of the services provided by the "Safeer" platform, and the second dimension about psychological alienation. The results of the study concluded that the reality of the digital platform " Safeer" has a high degree of supportive role during the first scholarship period for students. The results also showed that the reality of psychological alienation through the "Safeer" platform was moderate, for the two dimensions. This result indicates that the platform contributes to alleviating the psychological alienation of scholarship students. Accordingly, the study recommended developing a strategic plan to support communication by scholarship students with the digital platform " Safeer" through cultural attachés in countries of expatriate.

Keywords: The digital platform "Sphere" ; Psychological alienation; Scholarship.

\section{References:}

1. Al'bd Almn'm, F. (2017). Alm'wqat Aledaryh Alty Twajh Altlab Almbt'thyn Fy Dwlty Alwlayat Almthdh Alamrykyh Walmmlkh Almthdh Walsbl Almqtrhh Lltghlb 'lyha, Mjlt Drasat Trbwyh Wnfsyh: (94): 99-143.

2. 'qyl, '. (2017). Tjrbt Aldrash Balkharj W'laqtha Baldka' Althqafy Lda Mbt'thy Altrbyh Alkhash Mn Almmlkh Al'rbyh Als'wdyh. Almjlh Als'wdyh Lltrbyh Alkhash: (3) 1: 65-100.

3. Al'qyl, S. (2019). Almkhatr Alamnyh Almhtmlh Alta Qd Yt'rd Lha Almbt'thwn Als'wdywn Kharj Almmlkh Wsbl Alwqayh Mnha: Drash 'la 'ynh Mn Almbt'thyn Als'wdyyn Fa Amryka Wbrytanya Wastralya, Mjlt Aldrasat Alajtma'yh Als'wdyh: (2): 57-88.

4. Alasmry, '. (2019). Alsdmh Althqafyh W'laqtha Bb'd Almtghyrat Aldymwghrafyh Lda Altlbh Als'wdyyn Aldarsyn Fa Almmlkh Almthdh. Almjlh Als'wdyh Ll'lwm Alnfsyh: (64) 17: 99-119.

5. Alghamdy, Gh. (2016). Aldghwt Alnfsyh W'laqtha Balthsyl Alakadymy Lda 'ynh Mn Altlab Als'wdyyn Almbt'thyn Bjam't Awryqwn Balwlayat Almthdh Alamrykyh. Mjlt Alhkmh Lldrasat Altrbwyh Walnfsyh: (7): 8-33.

6. Alhdyby, A. (2014). Almshklat Alty Twajh Altlbh Als'dyyn Almbt'thyn Ela Alwlayat Almthdh Alamrykyh, Mjlt Jam't Shqra': (2) $4: 101-135$.

7. Alhlfawy, W. (2017). Nmwdj Mqtrh Lmnsh Fnyh 'br Alwyb Wqyas Fa'lytha Fy Tnmyt Altfkyr Alebda'y Lda Altlab Alm'lmyn Fy Altrbyh Alfnyh. Almjlh Almsryh Lldrasat Almtkhssh: (15) 8: 55-71.

8. Jdw', H. (2019). Dwr Shbkat Altwasl Alajtma'y Fy Tshkyl M'arf Wetjahat Almbt'thyn Als'wdyyn Tjah Alqdaya Almhlyh. Almjlh Al'rbyh Lbhwth Ale'lam Walatsal: (25): 346-396.

9. Kbajh, S. (2015). Altghyr Alqymy W'laqth Bhwyh Aldat Walaghtrab Alnfsy Lda Tlbh Althanwyh Al'amh Fy Qta' Ghzh. Rsalt Majstyr Ghyr Mnshwrh, Aljam'h Aleslamyh Bghzh. 
10. Aln'ym, '. (2015). Al'waml Alty Sa'dt Alftyat 'la Tfdyl Alabt'ath Ela Aldwl Almtqdmh, Drash Mtbqh 'la Almbt'that Als'wdyat Fy Alwlayat Almthdh Alamrykyh, Wastralya, Wbrytanya, Mjlt Sh'wn Ajtma'yh: (38): 121: 29-76.

11. Alrwyly, A. (2019). M'wqat Etmam B'thh A'da' Hy't Altdrys Als'wdyyn Fy Aljam'at Alajnbyh. Mjlt Sh'wn Ajtma'yh: (36): 930.

12. Alshryf, Sh. (2020). Waq' Atjahat Tlbh Aljam'h Nhw Twzyf Almnsat Alrqmyh Fy Alt'lym Aljam'y Balmmlkh Al'rbyh Als'wdyh. Mjlt Jam't Tybh Lladab Wal'lwm Alensanyh: (22): 352-406.

13. Al Alshykh, N. (2015). 'waml Tkyf Altalbh Almbt'thh: Drash Ttbyqyh 'la Almbt'that Fy Alwlayat Almthdh Alamrykyh. Almjlh Alajtma'yh: (10): 51-106.

14. Alsyd, M. (2018). Astkhdam Shbkat Altwasl Alajtma'y W'laqth Balaghtrab Alnfsy Lda Talbat Jam't Tybh. Mjlt Al'lwm Altrbwyh: (26) 3: 526-577.

15. Altmymy, N. (2019). Almshklat Alty Twajh Tlab Aldrasat Al'elya Almbt'thyn Llkharj Bjam't Alamyr Stam Bn 'bd Al'zyz. Mjlt Al'lwm Altrbwyh: (18): 419-474.

16. Wzart Alt'lym. (2020) . Tm Aletla' 1/2/1442 , Tm Estrja'h Mn Almsdr: Https://2u.Pw/Nodzc 